\title{
ERRATUM
}

\section{Low-cost mobile open-circuit hood system for measuring gas exchange in small ruminants: From manual to automatic recording}

\author{
C. FERNÁNDEZ, M. C. LÓPEZ AND M. LACHICA
}

DOI: doi.org/10.1017/S0021859615000416, published by Cambridge University Press, 11 May 2015

In the above mentioned article (Fernández et al. 2015), we regret to announce that Table 2 contained an incorrect footnote.

The correct version is supplied below.

Table 2. Daily energy $\left(\mathrm{kJ} / \mathrm{kg}^{0.75} \mathrm{BW}\right)$ and carbon-nitrogen $\left(\mathrm{g} / \mathrm{kg}^{0.75} \mathrm{BW}\right)$ balances, heat production (HP) and retained energy (RE) of female dry Manchega sheep $(n=12 ; 58 \pm 1 \cdot 2 \mathrm{~kg} B W$ as average; four sheep per diet) with the three offered diets based on cereal grain (CGR), fibrous by-products (FBP) and alfalfa hay $(A L H)$ calculated by indirect calorimetry ( $R Q$ method) and carbon-nitrogen balance ( $C N$ method)

\begin{tabular}{|c|c|c|c|c|c|}
\hline & CGR & $\mathrm{FBP}$ & $\mathrm{ALH}$ & S.E.M. & $P$ value \\
\hline Gross energy intake & 786 & 801 & 1010 & $35 \cdot 1$ & \\
\hline Energy in faeces & 212 & 214 & 445 & $34 \cdot 0$ & \\
\hline Energy in urine & 20 & 26 & 34 & $4 \cdot 2$ & \\
\hline Energy in methane & 40 & 51 & 58 & $2 \cdot 8$ & \\
\hline MEI & 514 & 511 & 474 & $14 \cdot 3$ & $0 \cdot 530$ \\
\hline \multicolumn{6}{|l|}{ RQ method } \\
\hline $\mathrm{HP}$ & 431 & 404 & 462 & $15 \cdot 5$ & $0 \cdot 046$ \\
\hline $\mathrm{RE}^{*}$ & 83 & 107 & 11 & $17 \cdot 8$ & $0 \cdot 041$ \\
\hline \multicolumn{6}{|l|}{ CN method } \\
\hline C intake & 18 & 18 & 26 & $1 \cdot 1$ & \\
\hline$C$ in faeces & $4 \cdot 2$ & $4 \cdot 4$ & $11 \cdot 1$ & 0.98 & \\
\hline$C$ in urine & 0.72 & $0 \cdot 50$ & $0 \cdot 80$ & $0 \cdot 054$ & \\
\hline $\mathrm{C}$ in $\mathrm{CO}_{2}$ & 10 & 10 & 11 & $0 \cdot 3$ & \\
\hline $\mathrm{C}$ in $\mathrm{CH}_{4}$ & 0.72 & 0.92 & $1 \cdot 05$ & $0 \cdot 051$ & \\
\hline$C$ retained & $2 \cdot 2$ & $2 \cdot 7$ & $1 \cdot 1$ & $0 \cdot 40$ & \\
\hline $\mathrm{N}$ intake & $1 \cdot 1$ & $1 \cdot 1$ & $1 \cdot 4$ & 0.05 & \\
\hline$N$ faeces & $0 \cdot 29$ & $0 \cdot 33$ & $0 \cdot 50$ & $0 \cdot 031$ & \\
\hline$N$ urine & $0 \cdot 43$ & $0 \cdot 32$ & $0 \cdot 33$ & $0 \cdot 031$ & \\
\hline$N$ retained & $0 \cdot 42$ & $0 \cdot 43$ & $0 \cdot 56$ & 0.056 & \\
\hline $\mathrm{RE}^{+}$ & 104 & 132 & 49 & $20 \cdot 2$ & $0 \cdot 011$ \\
\hline $\mathrm{HP}^{\ddagger}$ & 410 & 379 & 425 & $14 \cdot 4$ & $0 \cdot 011$ \\
\hline
\end{tabular}

S.E.M., standard error of mean; degrees of freedom $=2 ; \mathrm{MEl}$, metabolizable energy intake; $\mathrm{RQ}$, respiratory quotient; $\mathrm{CO}_{2}$, carbon dioxide; $\mathrm{CH}_{4}$, methane; $\mathrm{C}$, carbon; $\mathrm{N}$, nitrogen.

* Calculated as RE $=\mathrm{MEI}-\mathrm{HP}$.

+ Calculated as $\mathrm{RE}=51 \cdot 8 \times \mathrm{C}$ retained $-19 \cdot 4 \times \mathrm{N}$ retained

* Calculated as $\mathrm{HP}=\mathrm{MEI}-\mathrm{RE}^{\dagger}$. 


\section{REFERENCE}

C. FERNÁNDEZ, M. C. LÓPEZ and M. LACHICA. Low-cost mobile open-circuit hood system for measuring gas exchange in small ruminants: From manual to automatic recording. The Journal of Agricultural Science, available on CJO2015. doi.org/10.1017/S0021859615000416. 